\title{
Localization in a quantum spin Hall system
}

\author{
Masaru Onoda ${ }^{1,4}$ * Yshai Avishai ${ }^{2,3}$ † $^{\text {and Naoto Nagaosa }}{ }^{1,3,4}$ \\ ${ }^{1}$ Correlated Electron Research Center $(C E R C)$, \\ National Institute of Advanced Industrial Science and Technology (AIST), \\ Tsukuba Central 4, Tsukuba 305-8562, Japan \\ ${ }^{2}$ Department of Physics, \\ Ben-Gurion University , Beer-Sheva 84105 Israel \\ ${ }^{3}$ Department of Applied Physics, University of Tokyo, \\ Bunkyo-ku, Tokyo 113-8656, Japan \\ ${ }^{4}$ CREST, Japan Science and Technology Corporation (JST), \\ Saitama, 332-0012, Japan
}

\begin{abstract}
Localization problem of electronic states in a two-dimensional quantum spin Hall system (QSH - a symplectic model with a non-trivial topological structure) is studied by the transfer matrix method. The phase diagram in the plane of energy and disorder strength is exposed, and demonstrates "levitation" and "pair-annihilation" of the domains of extended states analogous to that of the integer quantum Hall system. The critical exponent $\nu$ for the divergence of the localization length is estimated as $\nu \cong 1.6$ which is distinct from both exponents pertaining to the conventional symplectic and the unitary quantum Hall systems. This strongly suggests a different universality class related to the non-trivial topology of the QSH system.
\end{abstract}

PACS numbers: $73.20 . \mathrm{Fz}, 72.25 .-\mathrm{b}, 73.43 .-\mathrm{f}, 03.65 . \mathrm{Vf}$

The spin Hall effect (SHE) in semiconductors, namely, generation of spin current perpendicular to an applied electric field, has recently attracted intensive attention following its theoretical proposals 1, 2, 3]. Recent experiments observed an accumulated spin polarization near the edges of a sample in two-dimensional n-type [4] and ptype [5] quantum wells of GaAs under an applied electric field. There still remain some controversial issues about the origin of these accumulated spins 6], i.e., whether it is due to extrinsic 7, 8, 9] or intrinsic [1, 3] origins. However, the fundamental importance of the SHE is the underlying topological structure of Bloch wavefunctions in systems with time-reversal (TR) symmetry. The concept of $\mathrm{SU}(2)$ non-Abelian Berry phase plays a crucial role in the intrinsic SHE 1, 2], which is a generalization of the anomalous Hall effect in metallic ferromagnets driven by the U(1) Berry phase [10]. These topological aspects led to the concept of spin Hall insulator (SHI) 11], which is a band insulator showing nonzero spin Hall conductivity. In these models 11], edge modes are shown to be gapful in generic cases 12], in sharp contrast with quantum Hall systems (see also ref. 13] where another proposal for the quantum spin Hall (QSH) system is discussed).

Originally, zero gap semiconductors such as $\mathrm{HgTe}, \alpha$ $\mathrm{Sn}$ and narrow gap semiconductors such as PbTe were listed as candidates for SHI 11. Later on, a model for QSH effect in graphene has been proposed 14, 15], which is shown to be topologically distinct from the previous ones since it is characterized by an odd number $Z_{2}$ of pairs of gapless edge modes for a semi-infinite system. In that case, Kramers theorem for systems with TR symmetry prevents the hybridization of edge modes with opposite chiralities 15, 16, 17]. The number $Z_{2}$ so defined is claimed to be related to the mod 2 index of the real Dirac operator and the latter is then explicitly constructed from the Bloch wavefunctions and used to distinguish the QSH phase from a simple SHI. Note however that spin is not a conserved quantity in the presence of spin-orbit interaction, and one cannot define a topological invariant of the first Chern class for the corresponding $\mathrm{SU}(2)$ gauge field 2]. (There is one proposal for the conserved spin current [18] which applies only for spatially homogeneous systems.) Therefore, spin Hall conductance is not related to a topological integer such as the $\mathrm{U}(1)$ Chern number $C_{\mathrm{U}(1)}$ for the charge quantum Hall effect (QHE) [19]. In this context, a recent work introduces a spin Chern number $C_{s c}$ (and a Chern number matrix), and investigates its stability toward disorder and its relationship to edge modes [20, 21, 22]. An important consequence drawn therein is that even though there is no conserved spin current, one can still define a conserved topological number associated with twisted (spin-dependent) boundary conditions 23]. Here the analogy with the QHE is rather appealing.

A question of paramount interest is how the occurrence of these new topological quantities affect the localization/delocalization properties of wave functions in the presence of disorder. Although the standard symplectic ensemble exhibits a metal-insulator transition in twodimensions, here we encounter a symplectic system with a non-trivial topological structure. It is already known for the quantum Hall systems, i.e., the unitary case, that $C_{\mathrm{U}(1)}$ [19] associated with the conserved charge current protects the isolated extended states at some special energies [24], although the naive renormalization group (RG) study of the unitary universality class dictates the local- 
ization for any finite disorder strength in two-dimensions 25]. Since $C_{\mathrm{U}(1)}$ is defined as a response to twisted boundary conditions, existence of extended states is necessary for having a nonzero $C_{\mathrm{U}(1)}$. This topological integer cannot change continuously. Rather, it jumps when two extended states with opposite Chern numbers annihilate each other. The pertinent field theory is the nonlinear- $\sigma$ model with a topological term, which results in two-parameter scaling RG equations 24]. Assuming that $\mathrm{Z}_{2}$ classification or $C_{s c}$ is also associated with extended states, we expect a similar scenario for the localization problem in the symplectic model belonging to the non-trivial $\mathrm{Z}_{2}$ classification and/or with nonzero $C_{s c}$.

Thus, in this work, we investigate the localization problem for a symplectic model with a nontrivial topological structure, i.e., QSH system with odd $\mathrm{Z}_{2}$ and $C_{s c}=2$. Using the transfer matrix method, the phase diagram in the plane of disorder strength and energy is revealed, which manifests the features of levitation and pair annihilation of extended states similar to the unitary QHE case, albeit with finite energy width of the extended region. Finite size scaling analysis of the localization/delocalization transition yields an exponent $\nu \cong 1.6$ for the divergence of the localization length, which is distinct from both that of the symplectic $(\nu \cong 2.73)[26$ ] and that of the unitary QHE $(\nu \cong 2.33)$ 27] universality classes. This strongly suggests a new universality class of the localization/delocalization transition in the symplectic class, due to the existence of a non-trivial topological structure.

The model Hamiltonian we study is a generalization of that proposed for graphene by Kane and Mele [14, 15]:

$$
\begin{aligned}
H & =\sum_{\langle i j\rangle} c_{i}^{\dagger}\left[t+i \lambda_{\mathrm{R}}\left(\boldsymbol{\sigma} \times \hat{\boldsymbol{d}}_{i j}\right)_{z}\right] c_{j} \\
& +\sum_{\langle i j\rangle^{\prime}} c_{i}^{\dagger}\left(t^{\prime}+i \lambda_{\mathrm{SO}} \nu_{i j} \sigma_{z}\right) c_{j} \\
& +\sum_{i} c_{i}^{\dagger}\left(\lambda_{v} \eta_{i}+w_{i}+h_{x} \sigma_{x}\right) c_{i} .
\end{aligned}
$$

Here $c_{i}^{(\dagger)}$ is the spinor annihilation (creation) operator, $\boldsymbol{\sigma}$ is the set of the Pauli matrices, $t$ is the conventional hopping energy between nearest neighbor sites $\langle i j\rangle$ (we use $t=1$ as the unit of energy), $\lambda_{\mathrm{R}}$ is the Rashba spin-orbit interaction strength and $\hat{\boldsymbol{d}}_{i j}$ is the unit vector connecting $\langle i j\rangle$. The constant $t^{\prime}$ is the conventional hopping energy between second nearest neighbor sites $\langle i j\rangle^{\prime}$. Here it is introduced just for assuring stability of the numerical analysis by the transfer matrix method and is fixed at the small value 0.01 . Moreover, $\lambda_{\mathrm{SO}}$ represents the spin-orbit interaction strength with $\nu_{i j}=(2 / \sqrt{3})\left(\hat{\boldsymbol{d}}_{1} \times \hat{\boldsymbol{d}}_{2}\right)= \pm 1$, where $\hat{\boldsymbol{d}}_{1}$ and $\hat{\boldsymbol{d}}_{2}$ are unit vectors along the two bonds connecting $\langle i j\rangle^{\prime}$, while $\lambda_{v}$ is the alternation of the site energies between the $\mathrm{A}$ and $\mathrm{B}$ sublattices $\left(\eta_{i}= \pm 1\right)$. The random potential $w_{i}$ is uniformly distributed between $-W / 2$ and $W / 2$. Finally, the last term represents the magnetic field along the $x$-direction, which breaks TR symmetry. The phase diagram of this model without the random potential $w_{i}$ and the magnetic field $h_{x}$ has been already displayed in the inset of Fig. 1 in ref. 14]. In the plane $\lambda_{v} / \lambda_{\mathrm{SO}}-\lambda_{\mathrm{R}} / \lambda_{\mathrm{SO}}$, there is a finite domain of QSH state with $Z_{2}=1, C_{s c}=2$, bounded by a curve where the gap closes. Outside this domain, the gap opens up again and the system becomes the usual SHI with $Z_{2}=0$, $C_{s c}=0$. Note that for $\lambda_{\mathrm{R}}=0$, the system is decoupled into two independent unitary subsystems [28, 29]. For $\lambda_{v}<\lambda_{v}^{c}$, each unitary model has $C_{\mathrm{U}(1)}$ 's of opposite signs for the valence and conduction bands, and the way of distribution of $C_{\mathrm{U}(1)}$ 's is opposite for each unitary model. Thus the total $C_{\mathrm{U}(1)}$ of valence bands is zero, but $C_{s c}=2$. In the case of $\lambda_{v}>\lambda_{v}^{c}, C_{\mathrm{U}(1)}$ 's of each band vanishes, and $C_{s c}$ is also zero. For finite $\lambda_{\mathrm{R}}$, these two unitary models are hybridized and become a symplectic model. $C_{s c}$ of this hybridized system is quantized as $C_{s c}=2$ in the domain of the QSH state, while it vanishes in the domain of the usual SHI. With a finite magnetic field $h_{x}$, similar hybridization occurs, but this breaks TR symmetry, and the model belongs to the topologicallytrivial unitary class where $C_{\mathrm{U}(1)}$ of each band is zero. Interestingly, in the clean limit, $C_{s c}$ of this unitary model is still quantized, i.e, $C_{s c}=2$, while each pair of edge states opens a gap due to the hybridization by $h_{x}$. (Note that $C_{s c}$ is well-defined even in TR breaking systems.)

The localization length $\lambda_{M}(W, E)$ of a quasi onedimensional tube of $M$-site circumference is calculated at energy $E$ and disorder strength $W$ by the transfer matrix method [30]. The $M$-dependence of the renormalized localization length $\Lambda_{M}(W, E)=\lambda_{M}(W, E) / M$ determines the localization/delocalization properties of the wavefunctions at energy $E$.

Figures 1(a-1)-(c-3) display $\Lambda_{M}(W, E)$ up to $M=24$ for several values of $W$ as functions of $E$ for disordered QSH system at $h_{x}=0$ (symplectic ensemble). The case $\lambda_{R}=0$ has been studied in the context of quantized anomalous Hall effect [29], and the two isolated extended states are identified by the $M$-independent $\Lambda_{M}(W, E)$. There are two energies at which extended states show up. As they merge together the extended states disappear. This is consistent with the scenario of levitation and pair annihilation of the two $C_{\mathrm{U}(1)}$ 's of opposite signs. With finite $\lambda_{R}$, the isolated extended states turn into finite energy region of extended states, the width of which increases with $\lambda_{R}$. Note that these two regions of extended states approach as the disorder $W$ increases. The gap in the density of states disappears already for $W$ larger than $\sim 3$, but still the two regions are separated. When $W$ is further increased, these two energy regions of extended states merge into one region, and eventually disappear. We have also checked that, in the ribbon geometry, there appear extended gapless edge states even when there are no extended bulk states in the middle energy region. Based on these results, we draw in Fig. 1(d) 

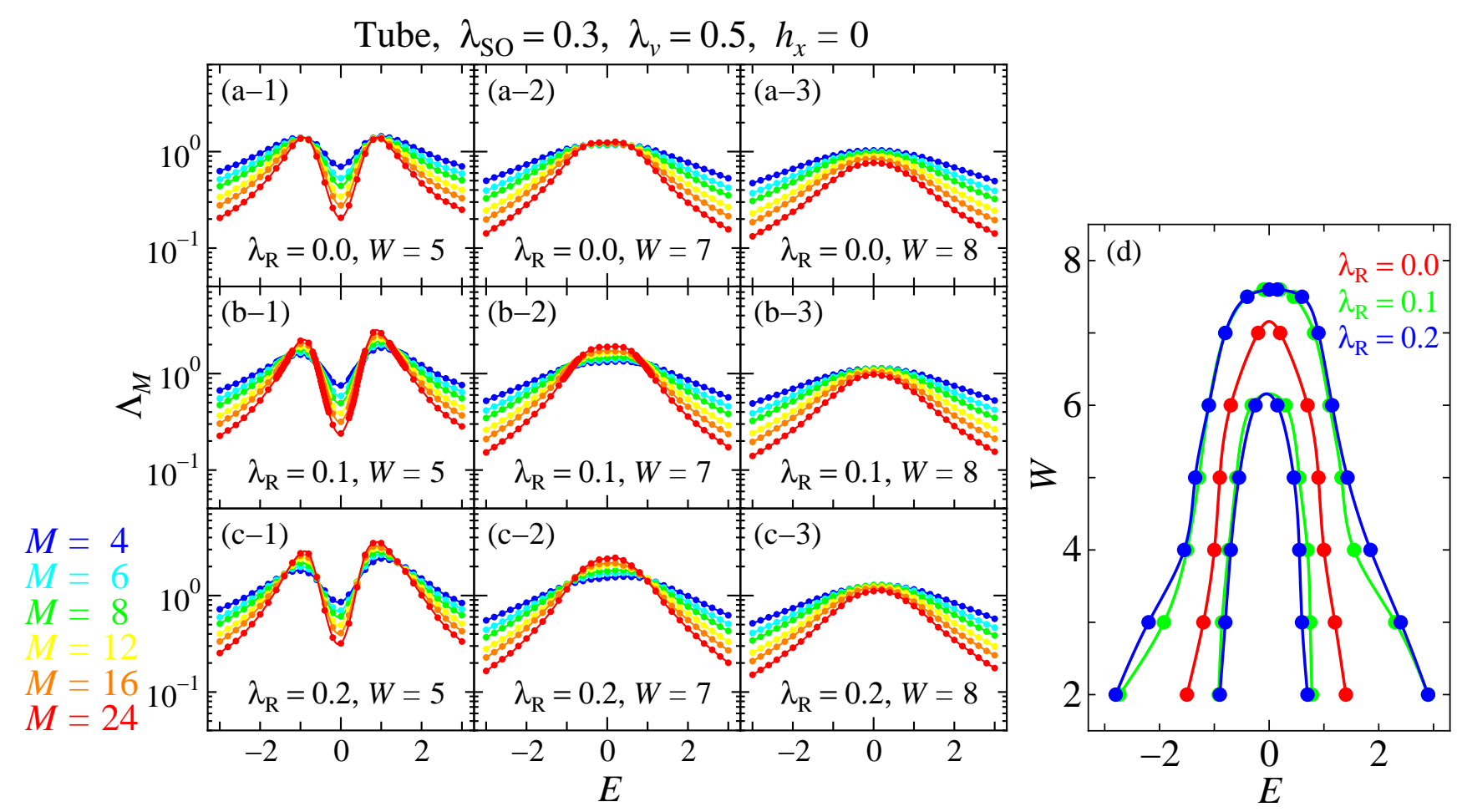

FIG. 1: Renormalized localization length $\Lambda_{M}(W, E)=\lambda_{M}(W, E) / M$ for QSH with $\lambda_{\mathrm{R}}=0(\mathrm{a}-1,2,3), \lambda_{\mathrm{R}}=0.1(\mathrm{~b}-1,2,3)$ and $\lambda_{\mathrm{R}}=0.2$ (c-1,2,3). The disorder strength $W$ is increased from left to right as $W=5.0(\mathrm{a}, \mathrm{b}, \mathrm{c}-1), W=7.0(\mathrm{a}, \mathrm{b}, \mathrm{c}-2)$ and $W=8.0$ (a,b,c-3). The other parameters are fixed as $\lambda_{\mathrm{SO}}=0.3, \lambda_{v}=0.5$ and $h_{x}=0$. (d) A localization/delocalization phase diagram obtained in the plane of energy $E$ and disorder strength $W$. The red curve is the energy of the isolated extended states for $\lambda_{\mathrm{R}}=0$, while the green and blue curves are the boundary of the energy region of the extended states for $\lambda_{\mathrm{R}}=0.1$ and $\lambda_{\mathrm{R}}=0.2$, respectively.

a phase diagram depicting the location of extended states in the $E-W$ plane. The red curve for $\lambda_{R}=0$ represents the trajectory of the isolated extended states in the unitary (QHE) case, while the phase boundaries between the localized and extended states are given by green curves for $\lambda_{R}=0.1$ and by blue ones for $\lambda_{R}=0.2$, respectively.

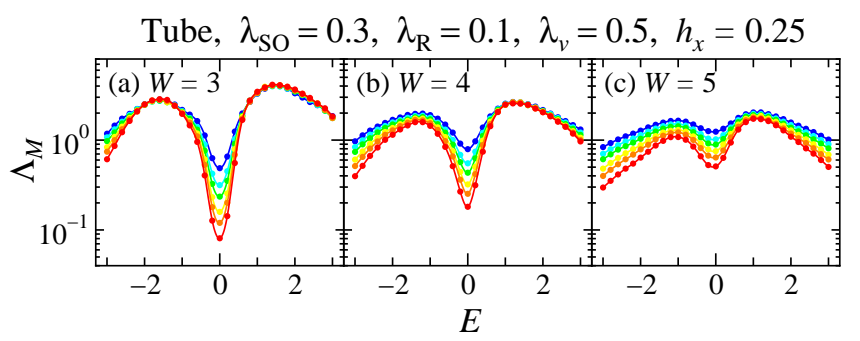

FIG. 2: Renormalized localization length $\Lambda_{M}(W, E)=$ $\lambda_{M}(W, E) / M$ for the unitary model with $h_{x}=0.25$ at (a) $W=3.0$, (b) $W=4.0$, (c) $W=5.0$. The other parameters are $\lambda_{\mathrm{SO}}=0.3, \lambda_{\mathrm{R}}=0.1$ and $\lambda_{v}=0.5$. All the states are already localized since the U(1) Chern numbers for up and down spin bands cancel out.

Let us confront some other cases with those discussed in Fig. 1] Figures 2(a)-(c) display the curves $\Lambda_{M}(W, E)$

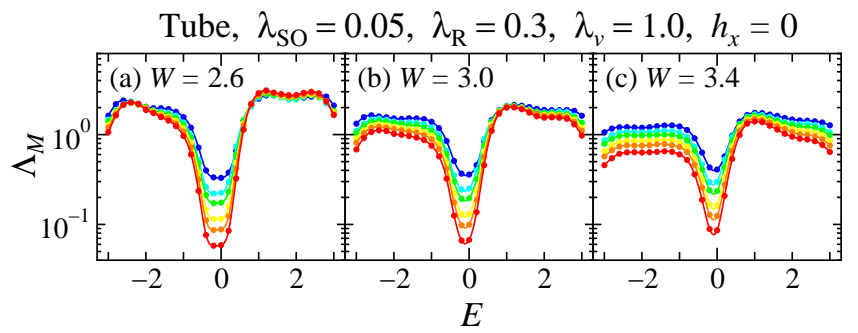

FIG. 3: Renormalized localization length $\Lambda_{M}(W, E)=$ $\lambda_{M}(W, E) / M$ for SHI with $\lambda_{\mathrm{SO}}=0.05, \lambda_{\mathrm{R}}=0.3$ and $\lambda_{v}=1.0$ at (a) $W=2.6$, (b) $W=3.0$ and (c) $W=3.4$.

for the unitary model with $h_{x}=0.25$ at (a) $W=3.0$, (b) $W=4.0$ and (c) $W=5.0$. In this case, the extended states have already disappeared since $C_{\mathrm{U}(1)}$ is zero for each of the split bands. Therefore the model is reduced to the trivial unitary class, where all states in two dimensions are localized with any finite amount of disorder [25]. (It is also confirmed that the gapful edge states in the ribbon geometry are localized.) However, it should be noted that $C_{s c}$ for this system is quantized as $C_{s c}=2$ in the clean limit. This means that, in TR breaking systems, finite $C_{s c}$ does not protect extended states and that 
protection would be closely related to the Kramers theorem. One might think that the difference is due solely to symmetry, i.e., unitary v.s symplectic classes, and not to the topological property of the QSH state. It is therefore important to compare with the simple symplectic model which belongs to the trivial $\mathrm{Z}_{2}$ classification. In our model, the SHI corresponds to this case, and Fig. 3 shows $\Lambda_{M}(W, E)$ for $\lambda_{S O}=0.05, \lambda_{R}=0.3, \lambda_{v}=1.0$ at (a) $W=2.6$, (b) $W=3.0$ and (c) $W=3.4$, respectively. It is evident here that the extended states disappear with much weaker disorder strength, and the two energy regions of extended states disappear without merging into a single one.

The above two cases, i.e., Figs. 2 and 3 strongly suggest that the localization behavior of QSH system in Fig. 1 is deeply influenced by the nontrivial topological aspect, and is distinguished from that of the usual symplectic class. In order to substantiate this expectation, we have studied the critical property of the localization/delocalization transition of the QSH system.

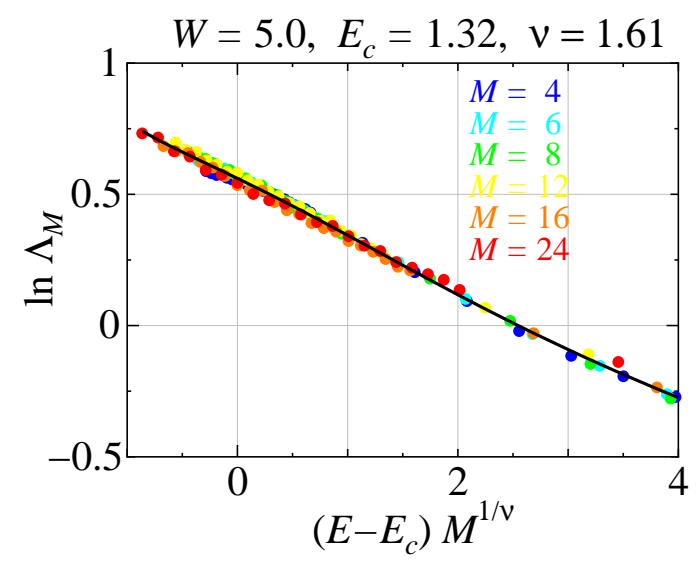

FIG. 4: Scaling plot of the renormalized localization length $\Lambda_{M}(W, E)$ (see raw data in Fig. 11 b-1)) as a function of $\mid E-$ $E_{c} \mid M^{1 / \nu}$ for different energies $E$ and $M \leq 24$ and for $W=5$. The critical energy and exponent are estimated as $E_{c}=1.32 \pm$ $0.005, \nu=1.61 \pm 0.10$, respectively.

Figure 4 summarizes the scaling analysis by displaying $\Lambda_{M}(W, E)=f\left(\left|E-E_{c}\right| M^{1 / \nu}\right)$ at $W=5.0$ with $\nu$ being the critical exponent for the divergence of the localization length. Data for various $E$ and $M$ (up to $M=24$ ) are included and their collapse on a single curve indicates a reasonable one parameter scaling behavior, which simultaneously determines $\nu=1.61 \pm 0.10$. We have also studied transition at higher disorder $W=7.0$, and found $\nu=1.61 \pm 0.10$. This exponent should be confronted with that of the standard symplectic universality class $\nu \cong 2.73$ [26], and that of the unitary model at strong magnetic field (with finite $\left.C_{\mathrm{U}(1)}\right) \nu \cong 2.33$ [27]. The result $\nu \cong 1.6$ obtained here is clearly distinct from both of these values, and suggests a new universality class for the symplectic ensemble with a non-trivial topological struc- ture. Intuitively, it is anticipated that the localization problem should be influenced by the finite spin Chern number. The construction of an effective field theory for this class is left for future investigations.

In summary, we have studied the localization/delocalization problem of the QSH system (a symplectic ensemble with non-trivial topological property) by the transfer matrix method. The phase diagram shows levitation and pair annihilation of the two energy regions of extended states, analogous to that of the unitary model with finite Chern number (the integer quantum Hall effect). The critical exponent $\nu$ for the localization/delocalization transition is estimated as $\nu \cong 1.6$, which is distinct from that of the standard symplectic universality class $(\nu \cong 2.73)$ and that of the unitary class with non-zero Chern number $(\nu \cong 2.33)$. This strongly suggests that the QSH system belongs to a new universality class characterized by a topological index such as the spin Chern number matrix or the $\mathrm{Z}_{2}$ index.

The authors would like to thank C. L. Kane, F. D. M.Haldane, S. C. Zhang and T. Ohtsuki for fruitful discussions. This work is financially supported by NAREGI Grant, Grant-in-Aids from the Ministry of Education, Culture, Sports, Science and Technology of Japan and JSPS long term program (YA).

* Electronic address: m.onoda@aist.go.jp

† Electronic address: yshai@bgu.ac.il

¥ Electronic address: nagaosa@appi.t.u-tokyo.ac.jp

[1] S. Murakami, N. Nagaosa, and S.-C. Zhang, Science 301, 1348 (2003);

[2] S. Murakami, N. Nagaosa, and S.-C. Zhang, Phys. Rev. B 69, 235206 (2004).

[3] J. Sinova, D. Culcer, Q. Niu, N. A. Sinitsyn, T. Jungwirth, and A. H. MacDonald, Phys. Rev. Lett. 92, 126603 (2004).

[4] Y. K. Kato, R. C. Myers, A. C. Gossard, and D. D. Awschalom, Science 306, 1910 (2004).

[5] J. Wunderlich, B. Kaestner, J. Sinova, and T. Jungwirth, Phys. Rev. Lett. 94, 047204 (2005).

[6] For a review see H. A. Engle, E. I. Rashba, and B. I. Halperin, cond-mat/0603306 and references therein.

[7] M. I. Dyakonov and V. I. Perel, Phys. Lett. A 35, 459 (1971).

[8] J. E. Hirsch, Phys. Rev. Lett. 83, 1834 (1999).

[9] S. Zhang, Phys. Rev. Lett. 85, 393 (2000).

[10] M. Onoda and N. Nagaosa, J. Phys. Soc. Jpn. 71, 19 (2002).

[11] S. Murakami, N. Nagaosa, and S.-C. Zhang, Phys. Rev. Lett. 93, 156804 (2004).

[12] M. Onoda and N. Nagaosa, Phys. Rev. Lett. 95, 106601 (2005).

[13] B. A. Bernevig and S.-C. Zhang, Phys. Rev. Lett. 96, 106802 (2006).

[14] C. L. Kane and E. J. Mele, Phys. Rev. Lett. 95, 146802 (2005). 
[15] C. L. Kane and E. J. Mele, Phys. Rev. Lett. 95, 226801 (2005).

[16] C. Xu and J. E. Moore, Phys. Rev. B 73, 045322 (2006).

[17] C. Wu, B. A. Bernevig, and S.-C. Zhang, Phys. Rev. Lett. 96, 106401 (2006).

[18] J. Shi, P. Zhang, D. Xiao, and Q. Niu, Phys. Rev. Lett. 96, 076604 (2006).

[19] D. J. Thouless, M. Kohmoto, M. P. Nightingale, and M. den Nijs, Phys. Rev. Lett. 49, 405 (1982).

[20] L. Sheng, D. N. Sheng, C. S. Ting, and F. D. M. Haldane, Phys. Rev. Lett. 95, 136602 (2005).

[21] D. N. Sheng, Z. Y. Weng, L. Sheng, and F. D. M. Haldane, cond-mat/0603054 (2006).

[22] X. L. Qi, Y. S. Wu, and S.-C. Zhang, cond-mat/060407 (2006).
[23] Q. Niu, D. J. Thouless, and Y.-S. Wu Phys. Rev. B 31, 3372 (1985).

[24] A. M. M. Pruisken, The Quantum Hall Effect (SpringerVerlag, 1987) pp117.

[25] P. A. Lee and T. V. Ramakrishnan, Rev. Mod. Phys. 57, 287-337 (1985).

[26] Y. Asada, K. Slevin and T. Ohtsuki, Phys. Rev. Lett. 89, 256601 (2002).

[27] B. Huckestein, Rev. Mod. Phys. 67, 357 (1995).

[28] F. D. M. Haldane, Phys. Rev. Lett. 61, 2015 (1988).

[29] M. Onoda and N. Nagaosa Phys. Rev. Lett. 90, 206601 (2003).

[30] A. MacKinnon and B. Kramer, Z. Phys. B 53, 1 (1983). 